\title{
Correlación entre niveles de ansiedad y el Trastorno por Déficit de Atención e Hiperactividad en niños de 6 a 8 años
}

\section{Correlation between anxiety levels and Attention Deficit Hyperactivity Disorder in Children from 6 to 8 years old.}

\author{
Alvarado, Johana* y Guijarro César \\ Carrera de Sicología Educativa y Orientación Vocacional \\ Universidad Católica de Cuenca, Extensión Cañar \\ Cañar, 030201, Ecuador \\ *jtalvaradoa@ucacue.edu.ec
}

\begin{abstract}
Resumen
En este trabajo investigativo se analiza la correlación existente entre los diferentes niveles de ansiedad y el Trastorno por Déficit de Atención e Hiperactividad (TDAH). La población estudiada corresponde a 85 niños y niñas que tienen en común el diagnóstico de TDAH y acuden a tratamiento psicológico en el Centro de Desarrollo Humano IDEAS, de la ciudad de Cuenca - Ecuador, de edades comprendidas entre los 6 y 8 años. Para la investigación se procedió al análisis y selección de casos diagnosticados con Trastorno por Déficit de Atención e Hiperactividad (TDAH), mediante la interpretación de los criterios diagnósticos del DSM-V y la revisión del historial personal de los participantes, en cuanto a los niveles de ansiedad se empleó el Cuestionario de Ansiedad Infantil (CAS). Se considera dentro del estudio el análisis de factores como la edad y género de los casos con TDAH. La interpretación de los resultados se desarrolló en base a estadísticos descriptivos y exploratorios, obteniendo una relación significativa entre el Trastorno por Déficit de Atención e Hiperactividad y los niveles de ansiedad media y alta, de igual manera se evidencia que hay una mayor proporción de niños con niveles de ansiedad y TDAH a diferencia de las niñas.
\end{abstract}

Palabras clave: Trastorno por Déficit de Atención e Hiperactividad,TDAH, ansiedad, niveles de ansiedad.

\begin{abstract}
This research work analyses the correlation between anxiety levels an Attention Deficit Hyperactivity Disorder (ADHD). The studied population was 85 children that were diagnosed with ADHD and attend to a Psychological treatment in the Desarrollo Humano IDEAS center, in Cuenca-Ecuador from 6 to 8 ages. For the investigation to carried out there was made an analysis and a selection of cases that were diagnosed with Attention Deficit Hyperactivity Disorder (ADHD) by means of interpretation of the DSM-V diagnosis criteria and the participants' personal background, related to the anxiety level there was applied a children's anxiety questionnaire. The children's age and gender are considered among the factors for the analysis of the study. The interpretation of the results was developed based on a descriptive and exploratory statistics, getting a significant relation between the medium and high anxiety levels and the Attention Deficit Hyperactivity Disorder, and in the same way it is evident that there is greater rate of children with anxiety level an ADHD than girls.
\end{abstract}

Key words: Attention Deficit Hyperactivity Disorder, anxiety, anxiety levels.

\section{Introducción}

El Trastorno por Déficit de Atención e Hiperactividad en sus siglas TDAH es uno de los trastornos más frecuentes en la población infantil, ya sea que se presente de manera aislada o se relacione con otros trastornos psicológicos. Es un trastorno infantil que presenta un impacto enorme en el medio personal del niño, pues afecta el área social, familiar y académica. Se trata de un trastorno del neurodesarrolllo, que según la (Association, 2015) se caracteriza por problemas significativos de inatención, conducta desorganizada, hiperactividad y/o impulsividad que interfiere en el funcionamiento de quien lo padece.
La ansiedad por otra parte es un estado emocional en donde se presenta angustia y desesperación, es una respuesta adaptativa ante las distintas amenazas, sean reales o imaginarias, que ayudan al organismo a prepararse para enfrentar situaciones de peligro. Al ser un estado emocional normal puede presentar variantes psicopatológicas, como los trastornos de ansiedad que tienen en común miedo $\mathrm{y}$ ansiedad excesivos junto a conductas que refuerzan el trastorno.

Los trastornos de ansiedad según el DSM-V se diferencian del miedo o la ansiedad normal propios del desarrollo por ser excesivos o en su defecto persistir más allá de 
los periodos de desarrollo apropiados, desarrollándose por lo general durante la infancia y persistiendo en el tiempo si no son tratados.

Ambos trastornos son considerados hoy en día como los trastornos más comunes en la infancia y adolescencia. Es así que si un niño presenta TDAH su diagnóstico suele ser sencillo; sin embargo cuando se presenta asociado a otro trastorno, como por ejemplo un trastorno de ansiedad, el diagnóstico diferencial es mucho más complicado, pues es más difícil determinar cuál de los dos es el trastorno que prevalece, o en ocasiones las manifestaciones ansiosas pasan desapercibidas.

En general los síntomas de ansiedad en niños con TDAH suelen ser leves y relacionarse con altos índices de estrés que el niño puede experimentar a lo largo de su desarrollo, por lo que al momento de intervenir, el especialista presta más atención al tratamiento de TDAH, pues al controlar este trastorno es más probable que la ansiedad disminuya progresivamente. Sin embargo existen situaciones en que la ansiedad en un niño con TDAH es muy intensa, puede presentar altos niveles de ansiedad generalizada, fobias variadas, conductas obsesivo-compulsivas y manifestaciones fisiológicas que complican su relación con el medio.

Por todo lo expuesto es importante abordar la relación presente entre el Trastorno por Déficit de Atención e Hiperactividad y la ansiedad, pues cada vez más se diagnostican casos de niños y niñas con TDAH, quienes manifiestan estados ansiosos que afectan sus relaciones interpersonales.

Pese a que se tratan de dos trastornos de etiología independiente, son patologías clínicas que se presentan en mayor proporción en la población infantil y juvenil, es así que (Ferrín, 2012) asegura que en uno de cada tres niños se presenta la correlación de TDAH con ansiedad, existiendo una relación directa, ya que los niños con TDAH son más propensos a presentar frustraciones y represiones que generan ansiedad y desmotivación para la realización de actividades personales. Igualmente se ha evidenciado que los niños con diagnóstico de ansiedad suelen presentar mayores problemas para concentrarse y fijar la atención en puntos centrales.

Por eso es preciso hablar de correlación y no de causalidad, que en palabras de (Gómez, 2006), manifiesta que son dos conceptos que tienden a asociarse pero son distintos, pues al hablar de variables correlacionadas como la ansiedad y el Trastorno por Déficit de Atención e Hiperactividad, no implica necesariamente que la una sea causa de la otra.

Según (Guerrero, 2014) los trastornos de ansiedad presentes en niños con TDAH suelen pasar desapercibidos, ya que en la mayoría de las ocasiones los padres no se dan cuenta que sus hijos presentan síntomas de ansiedad y confunden ciertas manifestaciones ansiosas como la preocupación anticipada ante algún peligro, la tensión muscular, intranquilidad, problemas para dormir, etc., como síntomas clásicos del TDAH, quedando la ansiedad como un trastorno enmascarado. Esta situación se ve reflejado no solo en las relaciones sociales y familiares del niño, sino que también se presenta a nivel escolar, generando graves consecuencias como bajo rendimiento académico, fracaso escolar, conductas inadecuadas, problemas emocionales, etc., características que se suman a los criterios propios de cada trastorno.

\section{Marco Teórico}

El Trastorno por Déficit de Atención e Hiperactividad, tiene su base en un modelo neuropsicológico propuesto por (C. López y Romero, 2014), quienes refieren que se trata de una triada con alteraciones en la atención, dificultad en el control de impulsos e inquietud psicomotriz. El TDAH se manifiesta por una deficiencia en la capacidad para inhibir y supervisar al mismo tiempo las conductas y por la dificultad para poner freno a las distracciones (déficit de atención), a los pensamientos y actividad (impulsividad) y a los movimientos y acciones constantes (hiperactividad.). (C. López y Romero, 2014) Es así que el TDAH puede ser considerado como un trastorno en el sistema ejecutivo, siendo difícil el control atencional, así como la ejecución de tareas dirigidas y la consecuente capacidad para la toma de decisiones, debido a un déficit en los niveles dopaminérgicos en el lóbulo frontal, especialmente en el área prefrontal, sin evidencia de lesión anatómica que justifique su déficit.

Este trastorno raramente se presenta de manera aislada, puede estar asociado con otros trastornos, los cuales son detectables a través del diagnóstico y evaluación psicológica y médica. Jensen (como se citó en (C. López y Romero, 2014) manifiesta que los niños con Trastorno por Déficit de Atención e Hiperactividad presentan altos niveles de comorbilidad tanto en psicopatología internalizante (ansiedad, depresión, trastorno bipolar, etc.) como externalizante (agresividad, comportamiento antisocial, problemas de conducta, etc.), dependiendo de la forma de expresión del TDAH así como del tipo predominante, es decir si predomina el Déficit de Atención o por el contrario domina la Hiperactividad y/o Impulsividad.

(J. López, Serrano, y Delgado, 2004) analizaron una muestra clínica, en la cual el $50 \%$ de casos con TDAH presentaban un trastorno internalizado con predominio de tipo ansioso, distribuidos en los siguientes porcentajes: $45 \%$ corresponde a ansiedad generalizada, $18 \%$ relacionado a ansiedad de separación, $15 \%$ a fobia simple, $10 \%$ a fobia social, $5 \%$ relacionado a trastorno obsesivo-compulsivo, $3 \%$ a depresión mayor y en último lugar con un $3 \%$ correspondiente a trastorno distímico y con una incidencia mayor en el sexo masculino.

De manera paralela (Díaz, 2006) expresa que existe una frecuente asociación entre el Trastorno por Déficit de Atención e Hiperactividad y los trastornos de ansiedad como ansiedad por separación, trastorno de angustia, trastorno obsesivo-compulsivo y ansiedad generalizada, de- 
mostradas a través de investigaciones epidemiológicas que indican que alrededor del $25 \%$ de los niños con Trastorno por Déficit de Atención e Hiperactividad presentan un trastorno de ansiedad, sin existir un vínculo etiopatogénico específico.

Al evaluar a niños con TDAH y comorbilidad ansiosa existen características específicas como excesiva preocupación por el fracaso y adelantamientos ansiosas ante sucesos futuros, por lo tanto son niños que requieren ser tranquilizados, además suelen tener un comportamiento miedoso y sufrir de alteraciones psicosomáticas, todas estas características la diferencian de aquellos casos con TDAH sin presencia de trastornos de ansiedad asociados. Se ha demostrado que existe un solapamiento de síntomas, en los cuales resulta complicado el determinar si un niño ha desarrollado TDAH a causa de la ansiedad, o si el TDAH puede generar conductas ansiosas, pues por lo general el niño ansioso suele mostrar estados de aprensión, dificultad de control y otros síntomas que confunden con el TDAH como inquietud, fatigabilidad, dificultad para concentrarse, irritabilidad, entre los más frecuentes (Artigas, García, y Rigau, 2013). De acuerdo a los estudios genéticos se refuerza la hipótesis que ansiedad y TDAH son trastornos independientes que se heredan por separado, pero que se potencian mutuamente, de esta manera se expresa la existencia de un diagnóstico dual.

Otra investigación desarrollada por (Pujota, 2012) menciona que existe una predisposición de los niños con TDAH para reaccionar a síntomas de tipo ansioso, lo cual repercute significativamente en sus relaciones interpersonales, y demuestran que mientras mayor sea el nivel de TDAH, mayor será el grado de ansiedad que presente, existiendo una correlación directa entre ambas variables.

En consecuencia es necesario llevar a cabo esta investigación para demostrar la relación existente entre estas dos variables y poder de esta manera abordar adecuadamente los trastornos. Es así que para la presente investigación se ha tomado como objetivo general el determinar la correlación existente entre los niveles de ansiedad y el TDAH en niños y niñas de 6 a 8 años que acuden al Centro de Desarrollo Humano IDEAS. Además se pretende indagar la distribución de los niveles de ansiedad según el género y edad de los participantes.

La investigación empleada es correlacional de tipo no experimental, mediante la utilización del Cuestionario de Ansiedad Infantil que evalúa la ansiedad manifiesta en niños con TDAH previamente diagnosticados.

\section{Metodología}

La investigación a realizarse es correlacional que según (Hernández, Fernández, y Baptista, 2010) relaciona dos o más variables, ideas o conceptos dentro de una investigación.

Las variables a emplear son el Trastorno por Déficit de Atención e Hiperactividad y la ansiedad presente en niños y niñas que acuden al Centro de Desarrollo Humano IDEAS, de la ciudad de Cuenca. El diseño de la investigación es de tipo no experimental pues las variables empleadas no han sido manipuladas para su presentación, ya que se pretende observar las variables tal como se presentan en su contexto natural para luego analizarlas (Hernández y cols., 2010).

(Hurtado, 2000) al referirse a población manifiesta que se trata del conjunto de seres que comparten características comunes a ser evaluadas dentro de una investigación, mediante el análisis de las variables planteadas para el caso. En cuanto a la población a investigar se trabajó con 85 niños y niñas, previamente diagnosticadas con Trastorno por Déficit de Atención e Hiperactividad, de edades comprendidas entre 6 y 8 años, que acuden al Centro de Desarrollo Humano "IDEAS" de la ciudad de Cuenca, provincia Azuay, país Ecuador.

(Vélez, 2001) afirma que una técnica es la habilidad requerida para realizar una acción e implica el conocimiento empírico de cómo ejecutarlo. Para el desarrollo de la investigación se procede a emplear la observación directa, que permita la identificación de casos de TDAH, junto con el análisis y revisión de las historias clínicas de los niños que acuden al Centro de Desarrollo Humano IDEAS.

Un instrumento es cualquier recurso que emplea el investigador para obtener la información del fenómeno estudiado (Sabino, 2014). Para la evaluación de este trabajo investigativo se emplea el Cuestionario de Ansiedad Infantil (CAS) que evalúa el nivel de ansiedad manifiesta. Para el procesamiento de la información se empleó estadísticos descriptivos y exploratorios aplicados a las variables de edad y género, mediante el programa informático SPSS, el mismo que permite analizar a su vez la validez y confiabilidad de la prueba psicométrica empleada.

\section{Resultados}

Es necesario mencionar que para llevar a cabo esta investigación todos los 85 casos analizados tenían en común el diagnóstico de Trastorno por Déficit de Atención e Hiperactividad (TDAH) de acuerdo a los criterios diagnósticos del DSM-V. En la tabla 1correspondiente al género de los estudiantes analizados se observa que presentan un promedio de 1,47 con una desviación típica de 0,50 y una mediana de 1 . Se puede evidenciar que existe un porcentaje de $53 \%$ correspondiente al sexo masculino y con un $47 \%$ para el género femenino, constatando que existe mayor número de niños con TDAH como indica el gráfico 1.

De la información de la tabla 2 se desprende que los niños y niñas con TDAH que acuden al Centro de

Tabla 1

Porcentaje Género

\begin{tabular}{lccrr}
\hline GÉNERO & Frecuencia & Porcentaje & \multicolumn{2}{c}{$\begin{array}{l}\text { Porcentaje } \\
\text { acumulado }\end{array}$} \\
\hline Masculino & 45 & 53 & 53 \\
Femenino & 40 & 47 & 100 \\
\hline Total & 85 & 100 &
\end{tabular}




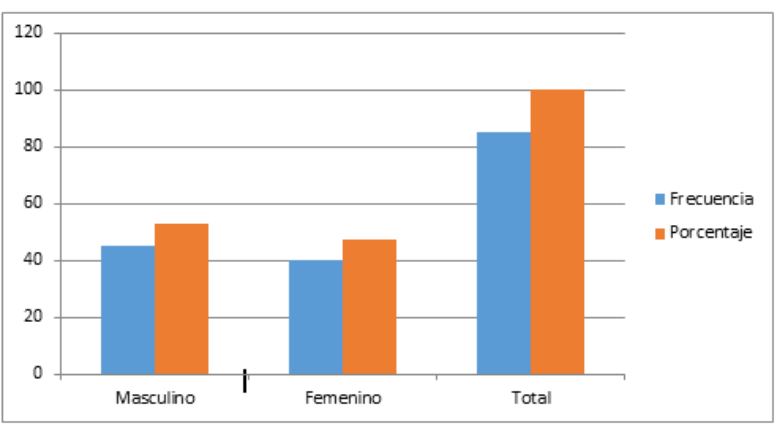

Figura 1. Género en estudiantes

Desarrollo Humano IDEAS tienen una edad promedio de 2.19, con una variabilidad de 0,55. La edad de los niños y niñas está ubicada en un rango de 2 años. En base a esto los niños y niñas con TDAH presentan una edad promedio de 7 años con un porcentaje de $67 \%$, continua en menor porcentaje aquellos niños que tienen 8 años con un $26 \%$ y en último lugar se encuentran los niños de 6 años con un porcentaje de $7 \%$.

Tabla 2

Frecuencia de Género en Estudiantes

\begin{tabular}{lcr}
\hline & EDAD & \\
\hline & $N$ & Válidos \\
Media & Perdidos & 0 \\
Mediana & & 2,19 \\
Moda & & 2 \\
Desv. típ. & & 2 \\
\hline
\end{tabular}

Para establecer la correlación entre los niveles de ansiedad y el TDAH se procedió a analizar las variables de género y edad con el puntaje total del cuestionario de ansiedad infantil (CAS), obteniendo que para género la correlación de Pearson tiene un puntaje de 0,06 , en cuanto a edad la correlación es de 0,15 y para el puntaje total tiene una equivalencia de 1 , demostrando de esta manera que los resultados son significativos y por tanto existe correlación entre los mismos. (Tabla 3)

De acuerdo a la prueba de Chi cuadrado se observa que según la equivalencia del CAS los niños con TDAH presentan un grado de ansiedad baja de 0,12 en cuanto a la ansiedad media se obtiene un valor de 0,54 y para el nivel de ansiedad alta se obtiene un puntaje de 0,40. De acuerdo a estos resultados se puede interpretar que la mayoría de los niños diagnosticados con TDAH presentan niveles de ansiedad media a alta, estableciéndose una relación significativa entre TDAH y los niveles de ansiedad. (Tabla 4)

En cuanto al grado de confiabilidad y validez de la prueba empleada para medir los niveles de ansiedad se analizó mediante el Alfa de Cronbach obteniendo
Tabla 3

Correlación entre Género y Edad

\begin{tabular}{|c|c|c|c|c|}
\hline & & GÉNERO & $\mathrm{DAD}$ & EQUIVALENCIA \\
\hline \multirow{3}{*}{ GÉNERO } & $\begin{array}{l}\text { Correlación de } \\
\text { Pearson }\end{array}$ & 1 & $-0,02301437$ & 0,062800301 \\
\hline & Sig. (bilateral) & & 0,83439516 & 0,568012192 \\
\hline & $\mathrm{N}$ & 85 & 85 & 85 \\
\hline \multirow{3}{*}{ EDAD } & $\begin{array}{l}\text { Correlación de } \\
\text { Pearson }\end{array}$ & $-0,02301437$ & 1 & 0,152294282 \\
\hline & Sig. (bilateral) & 0,83439516 & & 0,164096375 \\
\hline & $\mathrm{N}$ & 85 & 85 & 85 \\
\hline \multirow{3}{*}{ EQUIVALENCIA } & $\begin{array}{l}\text { Correlación de } \\
\text { Pearson }\end{array}$ & 0,0628003 & 0,15229428 & 1 \\
\hline & Sig. (bilateral) & 0,56801219 & 0,16409637 & \\
\hline & $\mathrm{N}$ & 85 & 85 & 85 \\
\hline
\end{tabular}

Tabla 4

Correlación entre Ansiedad y TDAH

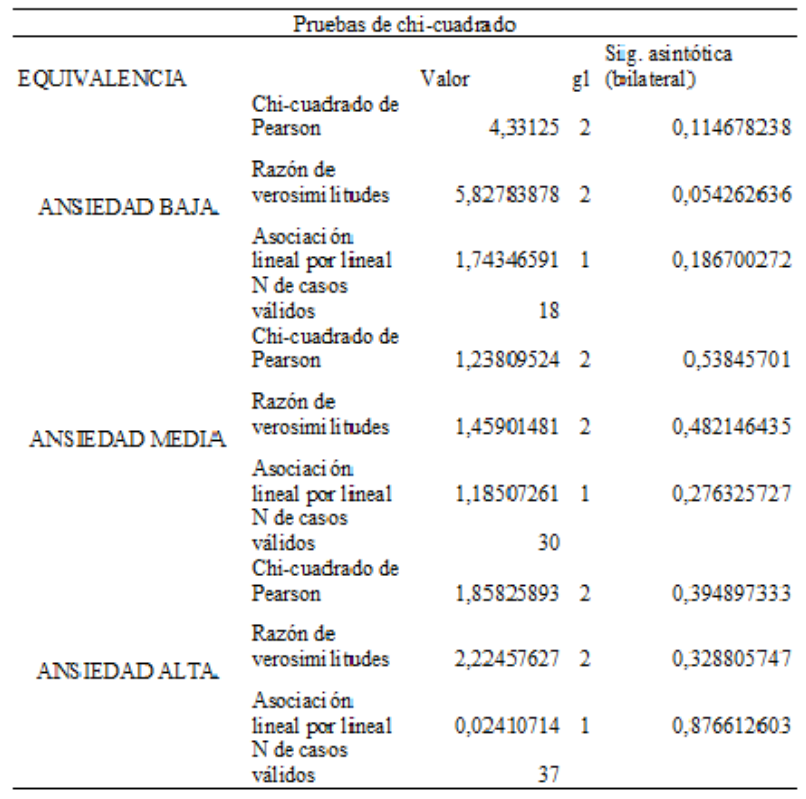

el valor de 0,7 que demuestra que el Cuestionario de Ansiedad Infantil es fiable para la investigación realizada. A continuación se detallan cada uno de los resultados obtenidos mediante el uso de estadísticos descriptivos y exploratorios del programa SPSS:

El estudio tenía como fundamento establecer la relación entre los niveles de ansiedad y el Trastorno por Déficit de Atención e Hiperactividad, de los resultados obtenidos se aprecia que el $67 \%$ de niñas y niños con TDAH presentan un nivel de ansiedad alta a la edad de 7 años, demostrando que se trata de un índice elevado de ansiedad, concordando con la conclusión obtenida por (Pujota, 2012), al manifestar que existe una tendencia marcada para reaccionar con síntomas ansiosos frente a un posible diagnóstico de Trastorno por Déficit de Atención e Hiperactividad. 
Se observa también que existe una mayor predisposición por parte del género masculino a presentar altos niveles de ansiedad, con un porcentaje del $53 \%$, debido a que el TDAH es más frecuente en niños que en niñas, por tanto los niveles serán más significativos en el sexo masculino.

En cuanto a la edad de los participantes y su relación con la presencia de ansiedad y TDAH, se manifiesta que la edad promedio es de 7 años, ya que la mayor parte de los encuestados se encuentran en esta edad de desarrollo, indicando que los varones presentan una puntuación mayor en ansiedad media a la edad de 7 años, a diferencia de las niñas que a la edad de 7 años presentan una ansiedad alta, esto se debe a que en muchos casos las diferencias personales, sociales y culturales influyen en la forma de adaptación de las personas a su entorno y por tanto determinan el comportamiento de las mismas. En cuanto a los niveles de ansiedad alta se observa que los niños y niñas a la edad 8 años presentan un punto de equilibrio.

Es necesario recalcar que a pesar de que los niveles de ansiedad parecen ser más elevados en el género femenino, se registró un mayor número de encuestados varones, por lo que los datos apuntan a que los niños con TDAH son más propensos a presentar problemas de ansiedad.

En cuanto a la correlación entre TDAH y niveles de ansiedad la prueba de Chi cuadrado establece una relación significativa, lo que indica que si existe una relación marcada entre ansiedad y TDAH, de esta manera se cumple el objetivo central de nuestra investigación. Lo que no se demuestra mediante esta investigación es que los niños con TDAH puedan presentar trastornos de ansiedad, así como que ansiedad y TDAH son resultado de la causalidad, ya que se tratan de dos trastornos con etiología propia, que pueden correlacionarse y presentarse de manera simultánea.

\section{Conclusiones}

- Según los resultados obtenidos se puede manifestar que los niños y niñas que se encuentran en las edades comprendidas entre 6 y 7 años, equivalente al nivel educativo de Segundo Año de Educación General Básica, muestran mayor predisposición a presentar niveles de ansiedad media a alta debido a la nuevas exigencias académicas que se presentan, es decir la presión por aprender a leer y escribir, esto se acentúa más en aquellos niños diagnosticados con TDAH, quienes presentar mayores dificultades de aprendizaje dadas por el trastorno en sí y por el medio educativo.

- Vale la pena aclarar que en base a las investigaciones analizadas y al estudio realizado no se da por sentado que el TDAH genere ansiedad o viceversa, sin embargo aquellos casos de TDAH son más susceptibles a presentar un grado mayor de ansiedad o su equivalente a algún tipo de trastorno.

- Si bien la investigación demuestra una relación significativa entre TDAH y los distintos niveles de ansiedad, es necesario realizar más investigaciones que demuestren si existen o no una comorbilidad entre TDAH y trastornos de ansiedad, ya que en muchos casos el trastorno de ansiedad puede presentarse enmascarado dentro del Trastorno por Déficit de Atención e Hiperactividad y generar mayores complicaciones en quien lo padece. Así como también es necesario llevar a cabo futuras investigaciones que tomen como referencia los diagnósticos de TDAH en función de sus variantes, es decir si existe predominio de inatención, hiperactividad o impulsividad, para poder compararlos y estudiarlos en relación a la presencia o no de manifestaciones ansiosas.

\section{Referencias}

Artigas, J., García, K., y Rigau, E. (2013). Comorbilidad en el tdah. En Ii congreso internacional de neuropsicología.

Association, A. P. (2015). Diagnostic and statistical manual of mental disorders. Autor.

Díaz, J. (2006). Comorbilidad en el tdah. Revista de Psiquiatría y Psicología del niño y el adolescente.

Ferrín, M. (2012). Tdah: hacia un consenso terapéutico. Actualización en Psiquiatría Infantil y Adolescente.

Gómez, M. (2006). Introducción a la metodología de la investigación científica. Editorial Brujas.

Guerrero, F. (2014). El lado oscuro del tdah en la edad adulta. Ediciones Aljibe.

Hernández, S., Fernández, C., y Baptista, L. (2010). Metodología de la investigación. Editorial McGrawHill.

Hurtado, J. (2000). Metodología de la investigación holística. Editorial Sypal.

López, C., y Romero, A. (2014). Tdah y trastornos del comportamiento en la infancia y en la adolescencia. Ediciones Pirámide.

López, J., Serrano, I., y Delgado, J. (2004). Trastorno por déficit de atención con hiperactividad: un modelo predictor de comorbilidad con trastornos del comportamiento. Revista Psicothema, 15(1).

Pujota, K. (2012). Estudio sobre la influencia de los niveles de los trastornos de déficit de atención e hiperactividad en el grado de ansiedad que presentan los niños y niñas de 6 a 10 años. (Tesis de Master no publicada). Universidad Central del Ecuador.

Sabino, C. (2014). El proceso de investigación. Editorial Episteme.

Vélez, C. (2001). Apuntes de metodología de la investigación. Universidad EAFIT.

Recibido: 30 de mayo de 2016

Aceptado: 05 de julio de 2017 
Johana Alvarado: Psicóloga Clínica, Docente a tiempo completo de la Universidad Católica de Cuenca, extensión Cañar, cursando maestría en Psicología Clínica en Universidad Peruana Cayetano Heredia. Autora del artículo Incidencia del Bullying en el aprendizaje de los Estudiantes de nivel medio de los establecimientos educativos de la parroquia central del Cantón Cañar. Autora del artículo Desarrollo lingüístico en los niños y niñas, CEI Ana Paredes de Alfaro del Cantón Cañar

César Guijarro: Psicólogo Clínico, Coordinador de la Universidad Católica de Cuenca, extensión Cañarcursando maestría en Psicología Clínica en Universidad Peruana Cayetano Heredia.

(Correo electrónico: caguijarror@ucacue.edu.ec) 